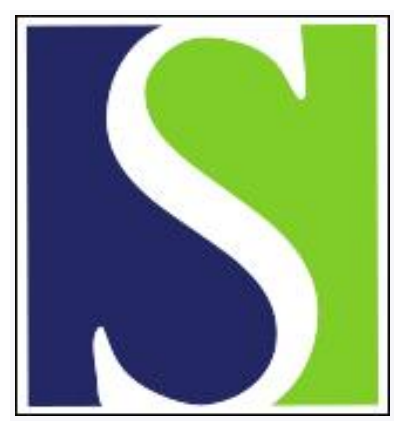

Scand J Work Environ Health 2012;38(4):343-348

https://doi.org/10.5271/sjweh.3267

Published online: 09 Jan 2012, Issue date: 01 Jul 2012

The relationship between current and former shift work and the metabolic syndrome

by Puttonen S, Viitasalo K, Härmä M

Affiliation: Finnish Institute of Occupational Health, Topeliuksenkatu 41 a A, FIN-00250, Helsinki, Finland. Sampsa.Puttonen@ttl.fi

Refers to the following texts of the Journal: 1997;23(4):257-265

2006;32(3):204-208 2008;34(3):198-205 2010;36(2):96-108

The following articles refer to this text: 2012;38(4):291-297;

2013;39(6):559-567; 2014;40(6):557-568

Key terms: cardiovascular risk factor; cardiovascular risk factor; epidemiology; metabolic syndrome; shift work

This article in PubMed: www.ncbi.nlm.nih.gov/pubmed/22231451 


\title{
The relationship between current and former shift work and the metabolic syndrome
}

\author{
by Sampsa Puttonen, PhD, ${ }^{1,2}$ Katriina Viitasalo, MD, ${ }^{3}$ Mikko Härmä, PhD ${ }^{1}$
}

\begin{abstract}
Puttonen S, Viitasalo K, Härmä M. The relationship between current and former shift work and the metabolic syndrome. Scand J Work Environ Health. 2012;38(4):343-348. doi:10.5271/sjweh.3267

Objective The occurrence of possible health hazards among former shift workers is not well-known. We studied associations of former and current shift work with the metabolic syndrome (MetS).

Methods Participants were 1811 full-time employees of a large airline company (1009 men). Working times were categorized into five groups: day worker [ $\mathrm{N}=297$ (the reference group)], former shift worker $(\mathrm{N}=341)$, 2-shift worker $(\mathrm{N}=418)$, night-shift worker $(\mathrm{N}=283)$, and in-flight worker $(\mathrm{N}=472)$. MetS was measured by the International Diabetes Federation (IDF) criteria and the National Institute of Health Adult Treatment Panel III (NCEP) guideline. The prevalence of the syndrome in the study population was $28.5 \%$ and $20.8 \%$, respectively.

Results Among male former shift workers, MetS was more prevalent compared to male day workers [IDF: age-adjusted odds ratio (OR) $2.13,95 \%$ confidence interval $(95 \% \mathrm{CI}) 1.35-3.37$; NCEP: OR 1.83, 95\% CI 1.13-2.96]. Associations did not change after additional adjustments for education, smoking, physical activity, alcohol consumption, and insomnia symptoms (IDF: OR 2.00, 95\% CI 1.26-3.19; NCEP: OR 1.67, 95\% CI 1.02-2.72). Male 2-shift workers also had an elevated risk of IDF-defined MetS (OR 1.64, 95\% CI 1.06-2.55) but the association weakened in the fully adjusted analyses (OR 1.48, 95\% CI 0.93-2.24). Prevalence of the MetS was marginally significantly higher among night-shift work (IDF: OR 1.51, 95\% CI 0.95-2.34) and was attenuated further with additional adjustments (OR 1.37, 95\% CI 0.84-2.22). Among women, no significant differences in prevalence of the MetS between day and shift work were observed.
\end{abstract}

Conclusion Findings of the cross-sectional study suggest that MetS diagnosed by standardized criteria is more prevalent among former male shift workers than current day workers who have never worked in shifts.

Key terms cardiovascular risk factor; epidemiology.

Shift work has become common in our $24 / 7$ society and around $20 \%$ of the European employees work in shifts, the corresponding number being, for example in China, as high as $36 \%$ (1). Epidemiological studies have repeatedly pointed to an increased risk of major diseases including cardiovascular diseases (CVD) in shift work (2). However, the pathways of the association and the specific groups of workers at risk are not fully understood. Shift work disturbs sleep and our natural biological body rhythms, and it may increase psychosocial stress and predispose to physiological disturbances related to metabolic syndrome (MetS) and CVD (3).

MetS is a clustering of risk factors for CVD such as insulin resistance, hypertension, lipid abnormalities, and central obesity (4). Karlsson and co-workers (5) found that several risk factors of MetS - including obesity, elevated triglycerides, and low levels of high-density lipoprotein (HDL) cholesterol - clustered together more often among shift than day workers. Other studies suggest that the occurrence of MetS as defined by the International National Cholesterol Education Program-Adult Treatment Panel III (NCEP) criteria is higher in rotating shift work $(6,7)$. However, in the study by Sookoian et al (7), shift work was unrelated to the International Diabetes Federation (IDF)-defined MetS. Recent followup studies have added to our knowledge by showing that rotating shift workers have an elevated risk for develop-

\footnotetext{
1 Finnish Institute of Occupational Health, Helsinki, Finland.

2 Institute of Behavioural Sciences, University of Helsinki, Helsinki, Finland.

3 Finnair Health Services, Vantaa, Finland.
} 
ing MetS when IDF or NECP MetS criteria is used as the outcome measure (8-10).

Despite previous reports suggesting that former shift workers and 2-shift workers may have an increased CVD risk $(11,12)$, previous MetS studies have concentrated on rotating shift work and therefore there is need to assess the risk in diverse shift schedules. In the present study, we separated former shift workers from current day workers and designated in-flight workers, whose work schedules are highly irregular, as a separate study group. Our aims were to study whether (i) the risk of MetS is increased among ex-shift workers, (ii) the previously reported relation between night-shift work and MetS can be repeated among employees of a large airline company (ie, Finnair), (iii) the occurrence of MetS is increased in types of shift work other than night shift, and (iv) there are gender differences in relation between working hours and MetS. We used two established MetS criteria in our study (IDF and NCEP) to evaluate the robustness of the associations and enhance comparability of results between studies.

\section{Methods}

\section{Participants}

During the years 2006-2008, one fifth of Finnair employees $(\mathrm{N}=4140)$ were invited for a check-up each year, altogether 2320 men and women attended. The check-up included physical examinations, laboratory tests, and questionnaires on working hours, sleeping habits, and lifestyles, in addition to counseling of an occupational health nurse or a doctor. Those 1811 participants, who were full-time workers and had complete data on all study variables, formed the final study group. They were 24-65 years old [mean age 44.3, standard deviation (SD) 8.9] and 1009 were male. The participation rates by age categories for men and women, respectively, were $40 \%$ and $60 \%$ ( $<35$ years), $50 \%$ and $60 \%$ (35-44 years), $57 \%$ and $68 \%$ ( $45-54$ years), and $56 \%$ and $62 \%$ ( $>55$ years). Of all participants, $40 \%$ were regular day workers, $39 \%$ non-flight shift workers, and $26 \%$ in-flight workers; in the target group, these percents were $33 \%, 39 \%$, and $28 \%$, respectively. The Ethics Committee of the Hospital District of Helsinki and Uusimaa approved the study and all subjects gave a written informed consent.

\section{Measures}

The participants were asked to indicate their current work schedule and whether they had previously worked in shifts. Working times were categorized into 5 groups: (i) day work (work done between 06.00-18.00 hours) (ii) former shift work (currently in day shift), (iii) 2-shift work (morning and evening shifts), (iv) night-shift work (3-shift work including night work $\geq 3$ hours between 23.00-06.00 hours), (v) in-flight work (irregular shift work with night work $\geq 3$ hours between 23.00-06.00 hours and/or time-lag $\geq 4$ hours). The two most frequent job types in the working time groups were as follows: (i) office work, planning, marketing (62\%), and aircraft service work (15\%); (ii) office work, planning, marketing (58\%), and managerial work (16\%); (iii) maintenance, storing work (30\%), and customer service (16\%); (iv) aircraft service work (51\%) and customer service (12\%); and (v) cabin crew (86\%) and pilots (11\%). Of the former shift workers, $65.0 \%$ had experience of night-shift work ( $\geq 1$ night shift per month); $48.2 \%$ of the 2 -shift workers were working regular morning and evening shifts and $30.5 \%$ reported working shifts that started before 06.00 hours $\geq 1$ per week. In night-shift work, the reported shift system was regular among $42.0 \%$ of employees and $53.2 \%$ on the regular shift system worked in a forward-rotating system. The majority of night-shift workers had either one $(30.8 \%)$ or three $(57.5 \%)$ consecutive night shifts. Work schedules in in-flight work were irregular (98.6\%) and $82.4 \%$ had trans-meridian flights (time-lag $>4$ hours).

The participant's educational level was measured by the highest education achieved categorized as $1=$ low (comprehensive school), 2=intermediate (secondary education) or $3=$ high (polytechnic or university). Physical activity was measured using a 4-choice question on weekly physical activity during leisure time (1=little/hardly any to $4=$ several times /competitive type). Alcohol consumption was measured as the number of $12 \mathrm{~g}$ units consumed per week. Smoking was coded as $1=$ smoker or $2=$ non-smoker.

Insomnia symptoms during the past three months were self-rated using a Likert-type scale $(1=$ never or $<1$ a month to $5=$ daily or almost daily) and the mean value of three questions ("How often have you had difficulties in initiating sleep?"; "How often do you wake up during the night?"; "How often do you feel tired during the day time?") was used as a continuous indicator of insomnia severity.

\section{Physiological measures and the MetS}

An occupational nurse or a physician carried out the check-up, which included measurements of blood pressure, height, weight, and waist circumference, and blood tests for lipids that were taken between 07:30-10:00 after a fasting period of 12 hours. In the invitation letter, the participants were urged not to come to the tests after a night shift. MetS according to the IDF criteria (13) was defined as central obesity (waist circumference $>80 \mathrm{~cm}$ and $>94 \mathrm{~cm}$ for women and men, respectively) plus any two of the following: (i) raised triglycerides 
$>1.7 \mathrm{mmol} / \mathrm{L}$, specific treatment for this lipid abnormality, (ii) reduced HDL-cholesterol $(1.03 \mathrm{mmol} / \mathrm{L}$ and $1.29 \mathrm{mmol} / \mathrm{L}$ among men and women, respectively), specific treatment for this lipid abnormality, (iii) raised systolic blood pressure $(>130 \mathrm{~mm} \mathrm{Hg})$, diastolic blood pressure $(>85 \mathrm{~mm} \mathrm{Hg})$, or treatment of previously diagnosed hypertension, and (iv) raised fasting plasma glucose $(>5.6 \mathrm{mmol} / \mathrm{L})$, or previously diagnosed type- 2 diabetes. MetS according to the NCEP guideline (14) was defined as the occurrence of $\geq 3$ of the following conditions: waist $>102 \mathrm{~cm}$ and $>88 \mathrm{~cm}$ among men and women, respectively; serum triglycerides $>1.7 \mathrm{mmol} / \mathrm{L}$ or treated; HDL-cholesterol $<1.03 \mathrm{mmol} / \mathrm{L}$ and $<1.3$ $\mathrm{mmol} / \mathrm{L}$ among men and women, respectively, or treated, systolic and diastolic blood pressure $>130$ or $>85 \mathrm{~mm}$ $\mathrm{Hg}$, respectively, or treated; and plasma glucose $>5.6$ $\mathrm{mmol} / \mathrm{L}$ or treated.

\section{Statistical analysis}

Analyses were done using SPSS version 17.0 (SPSS Inc, Chicago, IL, USA). The day worker group was set as the reference group, against which each of the other groups was contrasted. We used analysis of variance for the continuous variables and $\mathrm{chi}^{2}$ tests for discrete variables to test differences in control variables (age, sex, education, physical activity, alcohol consumption, smoking status, and insomnia) between the working time groups. Due to non-normal distribution, alcohol consumption was log-transformed prior to analyses. Next, we performed multiple logistic regression analysis to test the relationship between each control variable and the MetS. Finally, we calculated odds ratios (OR) and 95\% confidence intervals $(95 \% \mathrm{CI})$ for the risk of MetS in working time groups, with day work as the reference separately for men and women. The seven tested models were: (i) age-adjusted; (ii-vi) adjusted for age, and additionally for one of the other variables including education, physical activity, alcohol use, smoking, and insomnia; and (vii) fully-adjusted for all above covariates.

\section{Results}

The prevalence rates of the MetS were $28.5 \%$ for the IDF and $20.8 \%$ for the NCEP formulations. The mean age of day workers was 2.4 to 5.2 years higher than in other working time groups (P-values $<0.001$ ) and day workers reported less frequent insomnia compared to night-shift workers (P-value $<0.05)$ (table 1). The gender distribution was even in the day workers, but former-, 2-, and nightshift workers had more male than female participants, and, in the in-flight group, the majority of participants were female. The control variables were systematically associated with both formulations of the MetS (P-values $<0.001$ ) excluding insomnia, which was unrelated to MetS in the sample (P-values $>0.056$ ). The risk of MetS increased with age $(6 \%$ per year) and the risk was 2.4 (IDF) to 2.6 (NCEP) times higher among men compared to women. The risk of MetS among current smokers was $40 \%$ (IDF) to $60 \%$ (NCEP) higher than non-smokers. In addition, participants with low education level had a 1.8(IDF) to 2.1- (NCEP) fold risk for the prevalence of MetS compared to participants with high education.

Table 2 gives the results of comparisons between working time groups for the IDF-defined MetS, separately for men and women. Among men, former shift workers had a 2.13-fold (95\% CI 1.35-3.37) age-adjusted risk of IDF MetS. Further adjustments for other covariates, including education, smoking, physical activity, alcohol consumption, and insomnia symptoms, did not influence the results (full model: OR 2.00, 95\% CI 1.26-3.19). A similar, but slightly weaker association was observed for NCEP-defined MetS and former shift work among men (age-adjusted: OR 1.83, 95\% CI 1.13-2.96; fullyadjusted: OR 1.67, 95\% CI 1.02-2.72) (data not shown). The results also showed an association between 2-shift work and MetS (IDF: OR 1.64, 95\% CI 1.06-2.55) which attenuated still with further adjustments (full model: OR 1.48, 95\% CI 0.93-2.24) (table 2). However, the association for NCEP-defined MetS among male 2-shift workers was not significant (age-adjusted: OR 1.40, 95\% CI 0.89-2.22). Prevalence of the IDF-defined MetS was marginally significantly higher among night-shift work (age-adjusted: OR 1.51, 95\% CI 0.95-2.34) and the association attenuated further with additional adjustments (full model: OR 1.37, 95\% CI 0.84-2.22). Again, results of NCEP-defined MetS were weaker and non-significant (age-adjusted: OR 1.20, 95\% CI 0.73-1.96. In additional analyses, we compared the risk of former night-shift workers and other types of former shift workers with day workers and found that the risk in these two groups was of similar magnitude (age-adjusted associations among men: IDF, former night shift: OR 2.05, 95\% CI 1.24-3.36 and other type of former shift work: OR $2.32,95 \% \mathrm{CI}$ 1.26-4.30; NCEP, former night shift: OR $1.66,95 \% \mathrm{CI}$ 0.98-2.82 and other type of former shift work: OR 1.99 95\% CI 1.04-3.81). Among women, we did not observe significant differences in prevalence of the MetS between day work and shift work.

\section{Discussion}

Our study showed that among men, former shift worker is associated with the increased occurrence of IDF- and NCEP-defined MetS, a finding that needs to be confirmed in future studies. Also 2-shift working men had 
Table 1. Characteristics of the study sample $(\mathrm{N}=1811)$. [SD=standard deviation.]

\begin{tabular}{|c|c|c|c|c|c|c|c|c|c|c|c|c|c|c|c|}
\hline & \multicolumn{15}{|c|}{ Working time group } \\
\hline & \multicolumn{3}{|c|}{$\begin{array}{l}\text { Day work } \\
(\mathrm{N}=297)\end{array}$} & \multicolumn{3}{|c|}{$\begin{array}{l}\text { Former shift work } \\
\qquad(\mathrm{N}=431)\end{array}$} & \multicolumn{3}{|c|}{$\begin{array}{l}\text { 2-shift work } \\
(\mathrm{N}=418)\end{array}$} & \multicolumn{3}{|c|}{$\begin{array}{l}\text { Night-shift work } \\
\quad(\mathrm{N}=283)\end{array}$} & \multicolumn{3}{|c|}{$\begin{array}{l}\text { In-flight work } \\
(\mathrm{N}=472)\end{array}$} \\
\hline & Mean & $\mathrm{SD}$ & $\%$ & Mean & SD & $\%$ & Mean & SD & $\%$ & Mean & SD & $\%$ & Mean & SD & $\%$ \\
\hline Age & 47.7 & 8.9 & & 45.3 & $8.7^{\mathrm{a}}$ & & 45.0 & $8.7^{\mathrm{a}}$ & & 42.5 & $8.5^{\mathrm{a}}$ & & 42.9 & $8.7^{\mathrm{a}}$ & \\
\hline Sex (male) & & & 50.5 & & & $63.6^{a}$ & & & $68.2^{a}$ & & & $82.3^{a}$ & & & $26.3^{\mathrm{a}}$ \\
\hline Education (high) b & & & 55.6 & & & 44.3 & & & $21.8^{\mathrm{a}}$ & & & $23.0^{a}$ & & & $74.4^{\mathrm{a}}$ \\
\hline Physical activity (low) & & & 18.2 & & & 17.6 & & & 18.9 & & & 21.6 & & & $7.6^{\mathrm{a}}$ \\
\hline Alcohol use & 5.4 & 5.4 & & 6.4 & 7.8 & & $6.1^{\mathrm{a}}$ & 6.1 & & 5.7 & 5.5 & & 4.3 & $4.0^{c}$ & \\
\hline Smoking (yes) & & & 22.2 & & & 20.5 & & & 26.8 & & & 25.1 & & & $13.1^{\mathrm{c}}$ \\
\hline Insomnia & 2.47 & 0.83 & & 2.58 & 0.85 & & 2.56 & 0.83 & & 2.63 & $0.89^{c}$ & & 2.45 & 0.83 & \\
\hline
\end{tabular}

a Differs from day work group, $P \leq 0.001$.

b Polytechnic or university degree.

c Differs from day work group, $\mathrm{P}<0.05$.

Table 2. Working times and prevalence of International Diabetes Federation (IDF) metabolic syndrome (MetS). [OR=0dds ratio; 95\% $\mathrm{Cl}=95 \%$ confidence interval.]

\begin{tabular}{|c|c|c|c|c|c|c|c|c|}
\hline \multirow[t]{2}{*}{ Variables } & \multicolumn{2}{|c|}{$\begin{array}{l}\text { Day work versus } \\
\text { former shift work }\end{array}$} & \multicolumn{2}{|c|}{$\begin{array}{l}\text { Day work versus } \\
\text { 2-shift work }\end{array}$} & \multicolumn{2}{|c|}{$\begin{array}{l}\text { Day work versus } \\
\text { night-shift work }\end{array}$} & \multicolumn{2}{|c|}{$\begin{array}{l}\text { Day work versus } \\
\text { in-flight work }\end{array}$} \\
\hline & OR & $95 \% \mathrm{Cl}$ & $\mathrm{OR}$ & $95 \% \mathrm{Cl}$ & OR & $95 \% \mathrm{Cl}$ & $\mathrm{OR}$ & $95 \% \mathrm{Cl}$ \\
\hline \multicolumn{9}{|l|}{ Men } \\
\hline Age & 2.13 & $1.35-3.37$ & 1.64 & $1.06-2.55$ & 1.51 & $0.95-2.34$ & 1.21 & $0.70-2.01$ \\
\hline Age and education & 2.11 & $1.33-3.33$ & 1.57 & $0.99-2.48$ & 1.45 & $0.90-2.35$ & 1.23 & $0.71-2.13$ \\
\hline Age and physical activity & 1.87 & $1.20-2.93$ & 1.43 & $0.93-2.19$ & 1.11 & $0.71-1.74$ & 1.05 & $0.62-1.79$ \\
\hline Age and alcohol use & 2.05 & $1.29-3.24$ & 1.60 & $1.03-2.48$ & 1.52 & $0.95-2.41$ & 1.21 & $0.70-2.09$ \\
\hline Age and smoking & 2.12 & $1.34-3.34$ & 1.60 & $1.03-2.49$ & 1.47 & $0.93-2.34$ & 1.20 & $0.70-2.08$ \\
\hline Age and insomnia & 2.13 & $1.35-3.36$ & 1.64 & $1.06-2.54$ & 1.50 & $0.94-2.39$ & 1.21 & $0.70-2.09$ \\
\hline Full model a & 2.00 & $1.26-3.19$ & 1.48 & $0.93-2.24$ & 1.37 & $0.84-2.22$ & 1.33 & $0.76-2.33$ \\
\hline \multicolumn{9}{|l|}{ Women } \\
\hline Age & 1.31 & $0.74-2.32$ & 1.36 & $0.77-2.39$ & 1.12 & $0.48-2.60$ & 0.64 & $0.39-1.07$ \\
\hline Age and education & 1.32 & $0.74-2.36$ & 1.29 & $0.72-2.31$ & 1.18 & $0.50-2.77$ & 0.70 & $0.41-1.19$ \\
\hline Age and physical activity & 1.14 & $0.65-2.01$ & 1.10 & $0.63-1.92$ & 0.75 & $0.33-1.73$ & 0.57 & $0.34-0.94$ \\
\hline Age and alcohol use & 1.37 & $0.77-2.44$ & 1.40 & $0.79-2.47$ & 1.16 & $0.50-2.71$ & 0.65 & $0.39-1.09$ \\
\hline Age and smoking & 1.34 & $0.75-2.37$ & 1.35 & $0.77-2.38$ & 1.14 & $0.49-2.67$ & 0.68 & $0.41-1.13$ \\
\hline Age and insomnia & 1.28 & $0.72-2.27$ & 1.32 & $0.74-2.33$ & 1.07 & $0.46-2.51$ & 0.64 & $0.38-1.06$ \\
\hline Full model a & 1.51 & $0.83-2.77$ & 1.42 & $0.78-2.59$ & 1.31 & $0.54-3.17$ & 0.84 & $0.48-1.46$ \\
\hline
\end{tabular}

adjusted for age, education, physical activity, alcohol use, smoking, and insomnia symptoms.

a tendency for an increased prevalence of MetS, but the association disappeared with adjustment for insomnia, education, and health habits including smoking, drinking, and physical activity. Unlike some previous studies, MetS was not found to be more common among nightshift compared to day workers in the present sample of employees. We did not observe an increased prevalence of MetS among former female shift workers.

We had no data on individual reasons for moving into day work. Most of the former shift workers in the current sample $(65 \%)$ reported having done night-shift work earlier. However, we cannot estimate to what extent the higher prevalence of MetS in former shift workers can be attributed to possible direct effects of former night-shift work or to other work-related and non-work factors. However, health selection to day work is a plausible mechanism. Finnair systematically screens the health of its employees using its own occupational health services. As supported also by Finnish law, it is common that employees with health problems can move to less burdensome shifts. According to the occupational health physicians, the possibilities to move from shift to less strenuous work schedules have been rather good except for in-flight workers in the company. The costs to the employee usually are limited to reduced salary.

The possible health risks of 2-shift work are not well known. We found limited evidence for an elevated occurrence of MetS among male 2-shift workers. Most workers start shifts at 06.00 hours or later but very early shift starting times have become more common in the 
aviation business. Whether 2-shift work is associated with MetS and whether strenuous 2-shifts underlie the association remains to be explored in further studies. In addition, we tested if previous exposure to night shifts could explain the risk in 2-shift work but the results did not support this line of thought. Occurrence of the MetS was similar among participants with earlier experience of night shifts and those with no earlier night work (IDF among men: OR 1.64 and 1.65 , respectively).

The current study did not find a significant association between former shift work and MetS among women. The reason for this gender difference in occurrence of the MetS is not evident, however. The OR among women were in the same direction, but systematically lower compared to men. Thus, the association of former female shift workers and MetS reflects a general gender difference of the study. In addition, working conditions may vary between men and women, which can influence the observed differences. Male and female former shift workers may have moved to day work for different reasons and from different types of shifts making male and female groups different.

Unlike some previous studies (eg, 6, 7), we did not find a significant correlation between night-shift work and having MetS. The majority of the night-shift workers were aircraft maintenance workers, an occupational group which has adopted ergonomic shift systems in the company. An intervention study of the same employee group showed that the level of cardiovascular risk factors decreased and sleep, alertness, general health, wellbeing at work increased in this occupational group (15, 16). Thus it may be possible that both the systematic health screening of shift workers, as well as the implementation of ergonomic and flexible shift systems have influenced the risk in the present sample of night-shift workers. In addition, if we compare the observed OR for an increased risk in night work in our study with previously reported results, we see that the risk ratio is not exceptionally low. In the present sample, the number of participants in the night-shift group men was relatively small, which is reflected in the rather large confidence intervals.

In-flight workers showed no sign for an increased risk of MetS compared to day workers. They stood out from the other non-day work groups with respect to higher education level, better health behavior, and less insomnia symptoms. Also the strict aero-medical regulations influence their health, and their health is followed with frequent regular check-ups. Other studies show that despite their irregular working hours and jet-lag, flight attendants do not have a higher CVD or work-related cancer risk $(17,18)$. Our results are in line with this observation.

Using IDF criteria gave higher prevalence rates of MetS and also somewhat stronger associations with work schedules. Also, the risk ratio among former shift workers was somewhat higher for the IDF- compared to the NCEP-defined MetS, but in general findings for both international formulations were highly similar. Previous shift work studies have used either IDF or NCEP criteria to define MetS, with relatively similar findings for both. However, Sookoian and colleagues (7) found a significant association between rotating shift work and MetS defined by the IDF but not by NCEP criteria. Although both definitions appear to yield similar results, it is advisable to include more than one standard criterion to allow optimal comparisons between studies of shift work.

In our study, some limitations need to be raised. The study setting was cross-sectional, which does not allow conclusions about any time-dependent effects. This limitation concerns also our main result of former shift-workers. Although it is plausible that moving to day work and changes in the MetS status may both have been influenced by strenuous shift work, it is not possible to judge the association between former shift work and present signs of metabolic disturbances, largely due to non-accounted selection bias. Health selection into working time groups may have influenced present associations in general. In addition, the healthy worker effect may have influenced our results. The healthy worker effect states that those who stay in shift work are survivors who can withstand the strenuous shift work and that those who move into day work are likely to have a worse health status. Thus, the healthy worker effect may have resulted in an under- rather than overestimation of the effects of shift work. Kivimäki and co-workers (19) reported that employees with cardiovascular risk factors were more likely to leave an organization regardless of the type of work schedule. They concluded that health-related selection out of shift work is not a major source of bias in shift work and CVD studies. This is probably the case when employees do not have possibilities to move to day work in the same organization. Our results suggest that selection out of shift work can bias the results of shift work and cardiovascular risk factors when a high number of former shift workers are included in a group of day workers.

This is the first study to explore how various non-day work schedules and previous exposure to shift work are associated with MetS. Our results suggest that MetS is more prevalent among male former shift workers.

\section{Acknowledgement}

The Academy of Finland (SALVE consortium, grant 129518) and the Finnish Work Environment Fund (grant 108320) supported this study. 


\section{References}

1. Working time in the EU and other global economies. Luxembourg: Office for Official Publications of the European Communities; 2008.

2. Knutsson A. Health disorders of shift workers. Occup Med (Lond). 2003;53(2):103-8. http://dx.doi.org/10.1093/ occmed/kgg048.

3. Puttonen S, Härmä M, Hublin C. Shift work and cardiovascular disease - pathways from circadian stress to morbidity. Scand J Work Environ Health. 2010;26(2):96-108. http://dx.doi. org/10.5271/sjweh.2894.

4. Galassi A, Reynolds K, He J. Metabolic syndrome and risk of cardiovascular disease: a meta-analysis. Am J Med. 2006;119(10):812-9. http://dx.doi.org/10.1016/j. amjmed.2006.02.031.

5. Karlsson B, Knutsson A, Lindahl B. Is there an association between shift work and having a metabolic syndrome? Results from a population based study of 27,485 people. Occup Environ Med. 2001:58(11):747-52. http://dx.doi. org/10.1136/oem.58.11.747.

6. Esquirol Y, Bongard V, Mabile L, Jonnier B, Soulat JM, Perret B. Shift work and metabolic syndrome: respective impacts of job strain, physical activity, and dietary rhythms. Chronobiol Int. 2009;26(3):544-59. http://dx.doi. org/10.1080/07420520902821176.

7. Sookoian S, Gemma C, Fernandez Gianotti T, Burgueno A, Alvarez A, Gonzalez CD, et al. Effects of rotating shift work on biomarkers of metabolic syndrome and inflammation. J Intern Med. 2007;261(3):285-92. http://dx.doi.org/10.1111/ j.1365-2796.2007.01766.x.

8. De Bacquer D, Van Risseghem M, Clays E, Kittel F, De Backer G, Braeckman L. Rotating shift work and the metabolic syndrome: a prospective study. Int J Epidemiol. 2009;38(3):848-54. http://dx.doi.org/10.1093/ije/dyn360.

9. Pietroiusti A, Neri A, Somma G, Coppeta L, Iavicoli I, Bergamaschi A, et al. Incidence of metabolic syndrome among night shift health care workers. Occup Environ Med. 2009;67(1):54-7. http://dx.doi.org/10.1136/ oem.2009.046797.

10. Lin YC, Hsiao TJ, Chen PC. Persistent rotating shift-work exposure accelerates development of metabolic syndrome among middle-aged female employees: a five- year followup. Chronobiol Int. 2009;26(4):740-55. http://dx.doi. org/10.1080/07420520902929029.
11. Knutsson A, Hammar N, Karlsson B. Shift workers' mortality scrutinized. Chronobiol Int. 2004;21(6):1049-53. http:// dx.doi.org/10.1081/CBI-200035942.

12. Tenkanen L, Sjöblom T, Kalimo R, Alikoski T, Härmä M. Shift work, occupation and coronary heart disease over 6 years of follow-up in the Helsinki Heart Study. Scand J Work Environ Health. 1997;23(4):257-65. http://dx.doi.org/10.5271/ sjweh.218.

13. Alberti KG, Zimmet P, Shaw J. The metabolic syndrome--a new worldwide definition. Lancet. 2005;366(9491):1059-62. http://dx.doi.org/10.1016/S0140-6736(05)67402-8.

14. Grundy SM, Cleeman JI, Daniels SR, Donato KA, Eckel RH, et al. Diagnosis and management of the metabolic syndrome: an American Heart Association/National Heart, Lung, and Blood Institute Scientific Statement. Circulation. 2005;112:2735-52. http://dx.doi.org/10.1161/ CIRCULATIONAHA.105.169404.

15. Härmä M, Hakola T, Kandolin I, Sallinen M, Virkkala J, Bonnefond A, et al. A controlled intervention study on the effects of a very rapidly forward rotating shift system on sleepwakefulness and well-being among young and elderly shift workers. Int J Psychophysiol. 2006;59(1):70-9. http://dx.doi. org/10.1016/j.ijpsycho.2005.08.005.

16. Viitasalo K, Kuosma E, Laitinen J, Härmä M. Effects of shift rotation and the flexibility of a shift system on daytime alertness and cardiovascular risk factors. Scand J Work Environ Health. 2008;34(3):198-205. http://dx.doi.org/10.5271/sjweh.1228.

17. Pukkala E, Aspholm R, Auvinen A, Eliasch H, Gundestrup M, Haldorsen T, et al. Cancer incidence among 10,211 airline pilots: a Nordic study. Aviat Space Environ Med. 2003;74(7):699-706.

18. Houston S, Mitchell S, Evans S. Prevalence of cardiovascular disease risk factors among UK commercial pilots. Eur J Cardiovasc Prev Rehabil. 2011;18(3):510-17. http://dx.doi. org/10.1177/1741826710389417.

19. Kivimäki M, Virtanen $M$, Elovainio $M$, Väänänen A, Keltikangas- Järvinen L, Vahtera J. Prevalent cardiovascular disease, risk factors and selection out of shift work. Scand J Work Environ Health. 2006;32:(3):204-8. http://dx.doi. org/10.5271/sjweh.1000.

Received for publication: 2 May 2011 